\title{
Implications of Placebo and Nocebo Effects for Clinical Practice: Expert Consensus
}

\author{
Andrea W.M. Evers ${ }^{a}$ Luana Colloca ${ }^{b}$ Charlotte Blease ${ }^{c}$ Marco Annoni ${ }^{d}$ Lauren Y. Atlas $^{\mathrm{e}}$ \\ Fabrizio Benedetti ${ }^{f} \quad$ Ulrike Bingelg $^{g}$ Christian Büchel $^{\text {h }} \quad$ Claudia Carvalho $^{\mathrm{i}}$ Ben Colagiuri ${ }^{\mathrm{j}}$ \\ Alia J. Crumk ${ }^{k}$ Paul Enck' Jens Gaab ${ }^{m}$ Andrew L. Geers ${ }^{n}$ Jeremy Howick ${ }^{\circ}$ Karin B. Jensen $^{p}$ \\ Irving Kirsch ${ }^{q}$ Karin Meissner ${ }^{r}$ Vitaly Napadow ${ }^{\mathrm{s}}$ Kaya J. Peerdeman ${ }^{\mathrm{a}}$ Amir Raz $^{\mathrm{t}}$ \\ Winfried Rief $^{\mathrm{u}} \quad$ Lene Vase $^{\mathrm{v}}$ Tor D. Wagerw ${ }^{\mathrm{w}}$ Bruce E. Wampold ${ }^{\mathrm{x}, \mathrm{y}}$ Katja Weimer ${ }^{\mathrm{z}}$ \\ Katja Wiech ${ }^{\mathrm{A}}$ Ted J. Kaptchuk ${ }^{\mathrm{q}}$ Regine Klinger ${ }^{\mathrm{B}}$ John M. Kelley ${ }^{\mathrm{q}}$ \\ ${ }^{a}$ Health, Medical and Neuropsychology Unit, Institute of Psychology, Leiden University, Leiden, The Netherlands; \\ ${ }^{b}$ Departments of Pain Translational Symptoms Science and Anaesthesiology, School of Nursing and Medicine,

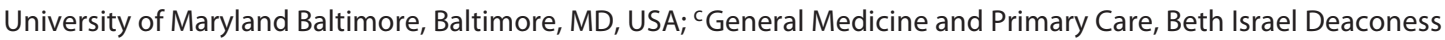 \\ Medical Center, Boston, MA, USA; ${ }^{\text {I}}$ Institute of Biomedical Technologies, National Research Council, Rome, Italy;

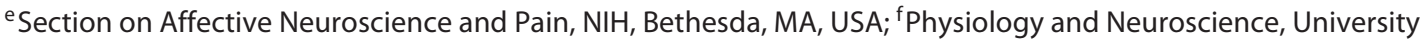 \\ of Turin Medical School, Turin, Italy; ${ }^{9}$ Department of Neurology, University Hospital Essen, Essen, Germany; \\ h Department of Systems Neuroscience, University Medical Center Hamburg-Eppendorf, Hamburg, Germany; \\ 'Department of Clinical and Health Psychology, Instituto Universitário de Ciências Psicológicas, Lisbon, Portugal; \\ jSchool of Psychology, University of Sydney, Sydney, NSW, Australia; ${ }^{\text {k}}$ Department of Psychology, Stanford \\ University, Stanford, CA, USA; 'Department of Psychosomatic Medicine and Psychotherapy, University Hospital \\ Tübingen, Tübingen, Germany; ${ }^{\mathrm{m}}$ Faculty of Psychology, University of Basel, Basel, Switzerland; ${ }^{\mathrm{n}}$ Department \\ of Psychology, University of Toledo, Toledo, OH, USA; ${ }^{\circ}$ Nuffield Department of Primary Care Health Sciences,

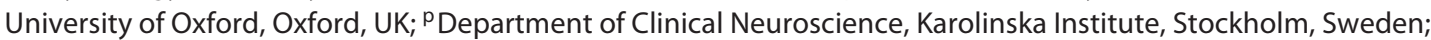 \\ ${ }^{\mathrm{a} B e t h}$ Israel Deaconess Medical Center, Program in Placebo Studies, Boston, MA, USA; ${ }^{r}$ Division of Health \\ Promotion, University of Applied Sciences, Coburg, Germany; ${ }^{5}$ Athinoula A. Martinos Center for Biomedical \\ Imaging, Charlestown, MA, USA; ${ }^{t}$ Departments of Psychiatry, Neurology and Neurosurgery, McGill University, \\ Montreal, QC, Canada; " Department of Clinical Psychology and Psychotherapy, Philipps University of Marburg, \\ Marburg, Germany; ${ }^{\vee}$ Department of Psychology and Behavioural Sciences, Aarhus University, Aarhus, Denmark; \\ ${ }^{w}$ Department of Psychology and Neuroscience, University of Colorado, Boulder, CO, USA; ${ }^{\times}$Department of \\ Counseling Psychology, University of Wisconsin-Madison, Madison, WI, USA; ${ }^{\mathrm{Y}}$ Modum Bad Psychiatric Center, \\ Vikersund, Norway; ${ }^{\mathrm{z} C l i n i c}$ for Psychosomatic Medicine and Psychotherapy, University Hospital Ulm, Ulm, Germany; \\ ${ }^{A}$ Nuffield Department of Clinical Neurosciences, University of Oxford, Oxford, UK; ${ }^{B}$ Center for Anesthesiology and \\ Intensive Care Medicine, University Medical Center Hamburg-Eppendorf, Hamburg, Germany
}

\section{Keywords}

Placebo effect - Nocebo effect · Patient's expectancies . Clinical practice - Patient-clinician communication .

Evidence-based ethical recommendations

\begin{abstract}
Background: Placebo and nocebo effects occur in clinical or laboratory medical contexts after administration of an inert treatment or as part of active treatments and are due
\end{abstract}

\begin{tabular}{ll}
\hline KARGER & $\begin{array}{l}\text { (c) } 2018 \text { The Author(s) } \\
\text { Published by S. Karger AG, Basel }\end{array}$ \\
E-Mail karger@karger.com & $\begin{array}{l}\text { This article is licensed under the Creative Commons Attribution- } \\
\text { NonCommercial-NoDerivatives 4.0 International License (CC BY- } \\
\text { NC-ND) (http://www.karger.com/Services/OpenAccessLicense). } \\
\text { Usage and distribution for commercial purposes as well as any dis- } \\
\text { tribution of modified material requires written permission. }\end{array}$
\end{tabular}

Andrea W.M. Evers

Health, Medical and Neuropsychology Unit, Institute of Psychology Leiden University, PO Box 9555

NL-2300 RB Leiden (The Netherlands)

E-Mail a.evers@fsw.leidenuniv.nl 
to psychobiological mechanisms such as expectancies of the patient. Placebo and nocebo studies have evolved from predominantly methodological research into a farreaching interdisciplinary field that is unravelling the neurobiological, behavioural and clinical underpinnings of these phenomena in a broad variety of medical conditions. As a consequence, there is an increasing demand from health professionals to develop expert recommendations about evidence-based and ethical use of placebo and nocebo effects for clinical practice. Methods: A survey and interdisciplinary expert meeting by invitation was organized as part of the 1st Society for Interdisciplinary Placebo Studies (SIPS) conference in 2017. Twenty-nine internationally recognized placebo researchers participated. $\boldsymbol{R e}$ sults: There was consensus that maximizing placebo effects and minimizing nocebo effects should lead to better treatment outcomes with fewer side effects. Experts particularly agreed on the importance of informing patients about placebo and nocebo effects and training health professionals in patient-clinician communication to maximize placebo and minimize nocebo effects. Conclusions: The current paper forms a first step towards developing evidence-based and ethical recommendations about the implications of placebo and nocebo research for medical practice, based on the current state of evidence and the consensus of experts. Future research might focus on how to implement these recommendations, including how to optimize conditions for educating patients about placebo and nocebo effects and providing training for the implementation in clinical practice.

(c) 2018 The Author(s)

Published by S. Karger AG, Basel

\section{Introduction}

Placebo and nocebo research has now evolved from a methodological consideration within clinical research to a distinct and expanding interdisciplinary field in its own right. Placebo studies encompass a broad variety of disciplines from biomedicine and neuroscience to the social and behavioural sciences and the humanities [1-14]. Placebo and nocebo effects refer to the beneficial or adverse effects that occur in clinical or laboratory medical contexts, respectively, after administration of an inert treatment or as part of active treatments, due to mechanisms such as expectancies of the patient. Current research incorporates both the study of placebo and nocebo responses as an outcome in clinical trials as well as the study of placebo and nocebo effects and their psychological and neurobiological mechanisms in various clinical applica-

Implications of Placebo and Nocebo Effects for Clinical Practice tions, including many medical disciplines. Current objectives in the field are to produce cross-disciplinary conceptualizations of placebo and nocebo effects; to re-evaluate the ethics of placebos in clinical trials and practice; and to initiate the ethical translation of empirical findings into clinical practice.

Robust empirical evidence now demonstrates that placebo and nocebo effects are both significant and measurable for many conditions (e.g., pain, depression, Parkinson's disease, fatigue, allergies, and immune deficiencies) [1]. Importantly, placebo and nocebo effects can substantially modulate the efficacy and tolerability of active pharmacological or other medical treatments $[15,16]$. This empirical evidence from experimental and clinical studies challenges health-care professionals to translate and implement the findings of placebo and nocebo research into practice. This translation relates not only to the possible use of placebos as part of regular treatments (e.g., placebo pills) but, far more importantly, to the systematic utilization of the mechanisms underlying placebo and nocebo effects to augment established treatment strategies (e.g., attention to expectations and empathy in patient-clinician communication).

Due to the interdisciplinary nature of this emerging field with its potentially wide applications across health care, there is a need to develop and formalize guidelines on the available evidence, including the possible implications of placebo and nocebo effects for clinical practice [17]. While the American Medical Association (AMA) provides explicit guidelines on the clinical use of placebos, the General Medical Council (GMC) in the UK provides no such ethical guidelines [18]. Notwithstanding the content of ethical guidelines and codes, studies reveal that the use of both pure (e.g., sugar pill) and impure (e.g., antibiotics for viral infections) placebos by doctors is widespread [19-21]: for example, in the US, 55\% of internists and rheumatologists reported using placebos; in the UK, $77 \%$ of primary care doctors reported that they used placebos at least once per week, while $86 \%$ of primary care doctors in Denmark admitted that they had used placebos at least once within the last year. Surveys aimed at investigating patients' attitudes reveal that the majority of respondents believe that placebo use by doctors is acceptable under certain circumstances [22]. Until now, however, there has been no study of expert opinions in placebo and nocebo studies about the implications of this research for clinical practice.

To address this gap, a clinical expert meeting was organized to survey established placebo researchers' views about placebo and nocebo effects and their translation 
into clinical practice. The aim of this survey and meeting was to develop a consensus on clinical recommendations based on the current state of the art in placebo and nocebo research among experts in the field.

\section{Methods}

\section{Expert Group}

Of the 31 invited speakers at the 1st official Society for Interdisciplinary Placebo Studies (SIPS) conference, 29 agreed to take part; the 2 who chose not to participate indicated that their research was not clinically focused. The 29 invited experts completed the survey, of whom 4 invited speakers were unavailable to participate due to personal circumstances. A workgroup of 6 members (A.W.M.E., L.C., C.B., R.K., T.J.K., and J.M.K.) prepared the survey and expert meeting.

The 29 participants were from 12 different countries, had an average age of 49.1 years (SD 11.1), and $45 \%$ were female. In total, $65.5 \%$ worked clinically (17.2\% physicians, $41.4 \%$ psychologists, and $6.9 \%$ acupuncturists). The participants' backgrounds included anaesthesiology, neurology, cognitive neuroscience, primary care, internal medicine, psychiatry, psychosomatic medicine, health and medical psychology, clinical psychology, epidemiology, medical ethics and philosophy. The participants had on average 14.2 years (SD 8.0) of research experience in the field of placebo and nocebo studies or related areas since their doctoral degree.

\section{Survey}

Based on a literature review of the relevant empirical evidence on possible clinical applications of placebo and nocebo effects, a survey was developed that focused on 4 themes: (a) prescription of placebo as regular treatment; (b) open-label prescription of placebo; (c) nocebo effects, and (d) patient-clinician communication. Subsequently, items for each theme were developed and checked by all members of the workgroup for relevance, readability and clarity. This process yielded 40 items in all, 10 for each theme. Participants were asked to read each statement and rank it on a 0 - to 10 -point scale with 0 meaning "totally disagree" and 10 meaning "totally agree." In addition, participants had the opportunity to provide written comments on each item (see online suppl. Appendix Table S1 for a complete overview of the survey; for all online suppl. Material, see www.karger.com/doi/10.1159/000490354). The survey was analysed by calculating the mean score for each item. High agreement with a statement was defined as a mean score $\geq 8$, and high disagreement with a statement was defined as a mean score $\leq 2$ on the 10 -point scale. Items that resulted in more mixed levels of agreement (scores between 2 and 8 ) were not discussed at the clinical expert meeting.

\section{Clinical Expert Meeting}

The clinical experts met in a 1-day pre-conference session in Leiden, The Netherlands, on April 2, 2017. At this meeting, the results of the survey and possible recommendations and discussion points were explored in plenary sessions. Consensus was reached based upon results of the survey and the discussion during the meeting. The meeting was audio-recorded and minutes were taken.
Table 1. Summary of the recommendations formulated by the expert group

Dos

1 Consider placebo effects as part of regular treatment

2 Inform patients about placebo and nocebo effects in such a way that treatment effects are maximized and side effects are minimized

3 Ensure a patient-clinician relationship that is characterized by trust, warmth and empathy in order to maximize placebo effects and minimize nocebo effects

4 Train health-care providers in patient-clinician communication to maximize placebo effects and minimize nocebo effects

5 Prefer open-label rather than hidden placebo prescription in those cases where there is evidence for efficacy and where prescribing a placebo is legal

Don'ts

1 Do not take risks (e.g., prescribing invasive treatments) to maximize placebo effects

2 Do not consider deception a necessary component of placebo effects

\section{Results}

In the sections below, we describe the main results of the survey and expert meeting. The initial discussion centred on defining important components of the placebo concept. We then summarize the survey statements with high agreement, as well as the related evidence, together with relevant comments discussed at the clinical expert meeting. A summary of the recommendations is listed in Table 1.

\section{Conceptualization of Placebo Effect and Placebo Response}

The experts agreed that it is crucial to distinguish between placebo and nocebo responses versus placebo and nocebo effects. In line with the drug terminology proposed by Fisher et al. [23] (1965), the placebo and nocebo response includes all health changes that result after administration of an inactive treatment (i.e., differences in symptoms before and after treatment), thus including natural history and regression to the mean. The placebo and nocebo effect refers to the changes specifically attributable to placebo and nocebo mechanisms, including the neurobiological and psychological mechanisms of expectancies. These mechanisms are shaped, for example, by 
verbal instruction, or nonverbal or situational cues that affect treatment expectancies (see communication by the SIPS community, July 29,2016 ) [see also 24]. Importantly, placebos and nocebos not only have effects during the prescription of placebo pills, but they can also substantially modulate the efficacy and tolerability of active pharmacological or other medical treatments. There was strong consensus that recommendations should be based on the research evidence on placebo and nocebo effects, including the involved mechanisms (e.g., expectancies) and their consequences for medical practice (e.g., patientclinician communication).

\section{Results of the Survey and Expert Meeting}

Considering Placebo Effects as Part of Regular

Treatment

There was a consensus regarding the importance of making optimal use of placebo effects to achieve better treatment outcomes (A1) and of optimally informing patients about placebo effects (A8), for example, to explain that a patient might improve due to factors other than the treatment itself, such as expectancies regarding treatment prognosis. The consensus was based primarily on the broad evidence that now exists for placebo and nocebo effects on clinical, neurobiological and laboratory outcomes, at least for the areas of pain, depression, Parkinson's disease, fatigue, allergies and immune deficiency [1-14]. However, the experts also acknowledged the current lack of knowledge about how best to provide this information to patients to ensure that patients are optimally informed. For example, there is insufficient knowledge about the ways in which patients and health-care providers can maximize placebo effects and minimize nocebo effects over time during repeated interactions and treatments [25].

The experts strongly agreed that clinicians should not prescribe or practice more invasive treatments simply to engender more potent placebo effects (A7). Although some systematic reviews have found that more intensive and invasive treatments can augment placebo effects, results are not consistent and are often moderated by factors such as the symptom or condition treated or the specific procedures used [26]. Moreover, there are clear practical and ethical restrictions on prescribing more invasive treatments for the sake of producing stronger placebo effects, since these procedures are often more expensive and entail a higher risk of undesirable side effects. Consequently, the experts agreed that prescription of more intensive and invasive treatment (e.g., injections instead of pills) to increase placebo effects is not justified.

Implications of Placebo and Nocebo Effects for Clinical Practice
Open-Label Placebo

Where open-label placebo (i.e., prescription of placebo pills with the knowledge and assent of the patient) was concerned, there was relatively high consensus that these should be preferred to hidden placebos (B3). Importantly, there are compelling ethical arguments for informing patients about placebo treatments in clinical contexts [27]. Related to these findings, the majority of the experts strongly agreed that deception is not necessary for placebo effects to occur (B2). This viewpoint is empirically supported by recent studies in patients with irritable bowel syndrome and chronic pain, which showed beneficial effects of open-label placebo on primary symptom outcomes, such as pain or disability [28-31]. However, more research is needed in other conditions and patient groups before recommendations can be developed about their possible use in clinical practice. For example, non-significant findings were found in a pilot study in patients with a major depressive disorder in a small sample of 12 patients, although the medium effect size was comparable to the other trials [32-34]. It has to be established whether specific patient groups or subgroups of patients benefit more than others from open-label placebos, or whether biological or psychological markers are associated with the effectiveness of these open-label placebo strategies. Promising is the case of dose-extending placebos (e.g., placebo given along with active medication) that mimic the psychobiological responses that are associated with the effectiveness of the medications with the potential of reducing side effects and costs [32]. Although prescription of placebos is not yet regulated in any part of the world and additional research is necessary on its shortand long-term effects, open-label prescription of placebos could be considered as a possible future watch and wait strategy or for use in long-term conditions when other treatment options have failed [33].

\section{Nocebo Effects}

There was a consensus regarding strategies to minimize and prevent nocebo effects. Experts agreed that nocebo effects should be explained to patients $(\mathrm{C} 1)$ and that information about side effects should be presented in such a way that nocebo effects are minimized (C4). The relatively strong consensus regarding optimally informing patients to minimize nocebo effects might be due to its high clinical relevance, which is based on findings that nocebo effects have been shown to consistently worsen treatment outcomes and to be at least in part responsible for side effects $[6,10-12,35]$. Indeed, there is convincing evidence that the way in which patients are informed 
about risks and side effects influences the likelihood of their occurrence. For example, when reassuring words are used during a local anaesthetic injection instead of emphasizing the pain experience during the procedure, pain reports can be reduced [36]. However, there are several ethical considerations that are required for optimal informed consent of patients, including balancing the need for honesty and transparency with the requirement that harms should not be induced or increased unnecessarily. For example, tailored strategies might be applied after the necessary information has been offered, depending on the patient's need for more detailed information on the risks and side effects of treatment. Such strategies might be particularly useful for those patients who have a high risk of developing nocebo effects, such as individuals who exhibit high levels of somatic amplification or fear of side effects $[7,10,11,35]$. Moreover, evidence is needed about the consequences of systematically informing patients about the role of nocebo effects, for example, by introducing the terminology of nocebo effects, or determining the optimal moment for informing patients about nocebo effects during ongoing treatments [37].

There was also consensus that clinicians should receive training and education to minimize nocebo effects (C8 and C9). Training and education might include informing health professionals about the negative impact of nocebo effects, as well as training them in strategies for optimal verbal and non-verbal communication, including information about risks and side effects [7, 9-11, 3537]. Again, however, there was acknowledgement that there has been a lack of research into the education and training of health professionals in relation to nocebo effects. Future studies might focus on evaluating different types of educational strategies to obtain insight into the most effective tools for training professionals to minimize nocebo effects (e.g., how information is communicated) as well as into optimal modes of education and training (e.g., including virtual reality tools).

\section{Patient-Clinician Communication}

The results of the survey showed a high level of agreement regarding patient-clinician communication (4 of 10 survey items). Experts agreed that a good patient-clinician relationship is essential to make optimal use of placebo effects to increase therapeutic efficacy (D2). Indeed, evidence is mounting that characteristics such as trust, warmth, and empathy are helpful in medical communication to foster placebo effects $[38,39]$. In addition, there was clear consensus that clinicians should receive regular education and training about how to make optimal use of placebo effects in their treatments (D6 and D7). However, studies examining which elements this training might include are scarce [17]. For example, a critical point in the patient-clinician communication is how to optimize expectancies without risking subsequent violation of patient's expectations and how to avoid eliciting overly positive expectations that might harm trust in the treatment. An important step will be to study specific features of disclosures (e.g., content, setting) with the aim of ethically tailoring education and training towards maximizing placebo and minimizing nocebo effects. Finally, experts agreed that medical ethics education encompassing placebo and nocebo effects should be a routine part of clinician training (D8).

\section{Discussion}

Intensive research in recent decades provides substantial evidence for the potential benefits of placebo effects, as well as the possible harms of nocebo effects [1-14]. In this survey of international experts on placebo research, there was strong consensus that patients should be informed about placebo and nocebo effects and that healthcare professionals should be trained to maximize placebo effects and minimize nocebo effects. The current paper forms a first step towards developing evidence-based and ethical recommendations about the implications of placebo and nocebo research for medical practice, based on the current state of evidence and the consensus of experts.

More research is needed in areas such as the prescription of open-label placebos, the optimization of intervention strategies to maximize placebo effects and minimize nocebo effects, and generalizability across different conditions. For example, it is important to ascertain which specific strategies for informing and educating patients and health professionals work best, as well as the optimal timing for the delivery of these interventions. It is also relevant to study the possible additive or interactive effects of different ways to maximize placebo effects, such as optimally informing patients, changing environmental cues or using conditioning during medical procedures. This might be particularly urgent in the area of nocebo effects, for example, for those patients who have a higher risk of developing severe adverse events due to high levels of fear of side effects or previous traumatic medical procedures. Large-scale research should also be encouraged with the aim to understand the substantial individual differences between placebo and nocebo responders and the possible neurobiological, psychological and genetic pre- 
dictors. In addition, both condition- and treatment-related predictors have to be taken into account (e.g., disease severity, length of the treatment) [1-14]. Finally, previous evidence for the context dependency of placebo effects emphasizes the need for further research into the interaction of stable predictors with situational factors [40].

This paper focuses on the possible clinical implications of placebo and nocebo effects. However, both placebo and nocebo effects as well as responses are key themes for further study in health research. For example, the probability of placebo assignments frequently contributes to the placebo response in clinical trials, showing larger effects in trials with a higher chance of getting the real treatment in comparison to the placebo conditions $[2,3,41$, 42 ]. The addition of a hidden or unpredictable prescription of the treatment might be a future path to minimize these placebo responses in clinical trials [2,3]. However, the development of clinical implications largely depends upon research on placebo and nocebo effects, based on the understanding of their underlying mechanisms and the development of innovative interventions. Importantly, these clinical implications involve placebo and nocebo effects upon administration of an inert substance or as an active treatment, since both are affected by comparable mechanisms, such as the patient's expectancies [1-5]. It is also important to emphasize that these recommendations cannot be generalized in a straightforward manner to non-medical contexts, such as psychotherapy [43]. Several relevant areas, such as the promising field of pharmacological conditioning, or predictors of placebo and nocebo effects, were omitted from the survey because, so far, the relevant evidence is only preliminary [1-14, 4446]. Finally, we did not focus on specific determinants of placebo and nocebo effects, such as cultural, ethnic, neurobiological or personal characteristics of patients, and the specific ethical and philosophical concerns that these factors raise.

Placebo and nocebo studies constitute a scientifically mature field of interdisciplinary research with applications in different medical disciplines and conditions. This burgeoning research calls for evidence-based recommendations for health professionals in medical practice. The initiative of the current survey and expert meeting is an important step towards the development of future guidelines aimed at helping medical clinicians and other health professionals to improve health care in their daily practice.

\section{Acknowledgment}

We would like to acknowledge the following colleagues for their support in the preparation of the survey and meeting described in this paper: G. Einthoven, J. Tekampe, D.S. Veldhuijzen, J.W.R. van Leusden, J.A.B. Mink and A.E. Breugem.

\section{Disclosure Statement}

The authors have no conflict of interest to disclose.

\section{Funding Sources}

This report was supported by an ERC Consolidator Grant, granted to A.W.M. Evers (ERC-2013-CoG 617700 EXPECT HEAL-TH); grants of the National Institutes of Health, granted to L. Colloca (NIDCR, R01DE025946) and granted to V. Napadow (NCCIH, R61-AT009306; Office of Director, OT2-OD023867); as well as an Irish Research Council/Marie Skłodowska Curie Global Fellowship, granted to C. Blease (CLNE/2017/226). The funders had no role in the acquisition or analysis of the data or the content of this article.

\section{References}

1 Kaptchuk TJ, Miller FG: Placebo effects in medicine. N Engl J Med 2015;373:8-9.

2 Enck P, Bingel U, Schedlowski M, Rief W: The placebo response in medicine: minimize, maximize or personalize? Nat Rev Drug Discov 2013;12:191-204.

3 Benedetti F: Placebo Effects. Understanding the Mechanisms in Health and Disease, ed 2. New York, Oxford University Press, 2015.

4 Colagiuri G, Schenk LA, Kessler MD, Dorsey SG, Colloca L: The placebo effect: from concepts to genes. Neuroscience 2015;307:171190.
5 Wager TD, Atlas L: The neuroscience of placebo effects: connecting context, learning and health. Nat Rev Neurosci 2015;16:403-417.

6 Schedlowski M, Enck P, Rief W, Bingel U: Neuro-bio-behavioral mechanisms of placebo and nocebo responses: implications for clinical trials and clinical practice. Pharmacol Rev 2015;67:697-730.

7 Evers AWM: Using the placebo effect: how expectations and learned immune function can optimize dermatological treatments. Exp Dermatol 2017;26:18-21.
8 Peerdeman KJ, van Laarhoven AIM, Keij SM, Vase L, Rovers MM, Peters ML, Evers AWM: Relieving patients' pain with expectation interventions: a meta-analysis. Pain 2016;157: 1179-1191.

9 Klinger R, Colloca, L, Bingel U, Flor H: Placebo analgesia: clinical applications. Pain 2015;155:1055-1058

10 Colloca L, Finniss D: Nocebo effects, patientclinician communication, and therapeutic outcomes. JAMA 2012;307:567-568.

11 Greville-Harris M, Dieppe P: Bad is more powerful than good: the nocebo response in medical consultations. Am J Med 2015;128:126-129.
Implications of Placebo and Nocebo Effects for Clinical Practice
Psychother Psychosom 2018;87:204-210 DOI: $10.1159 / 000490354$ 
12 Petersen GL, Finnerup NB, Colloca L, Amanzio M, Price DD, Jensen TS, Vase L: The magnitude of nocebo effects in pain: a meta-analysis. Pain 2014;155:1426-1434.

13 Klinger R, Kothe R, Schmitz J, Kamping S, Flor H: Placebo effects of a sham opioid solution: a randomized controlled study in patients with chronic low back pain. Pain 2017; 158:1893-1902.

14 Fava GA, Guidi J, Rafanelli C, Rickels K: The clinical inadequacy of the placebo model and the development of an alternative conceptual model. Psychother Psychosom 2017;86:332340.

15 Amanzio M, Pollo A, Maggi G, Benedetti F: Response variability to analgesics: a role for non-specific activation of endogenous opioids. Pain 2001;90:205-215.

16 Aslaksen PM, Zwarg ML, Eilertsen $\mathrm{HIH}$, Gorecka MM, Bjørkedal E: Opposite effects of the same drug: reversal of topical analgesia by nocebo information. Pain 2015;156:39-46.

17 Bishop GL, Coghlan B, Geraghty AWA, Everitt $\mathrm{H}$, Little $\mathrm{P}$, Holmes MM, Seretis D, Lewith G: What techniques might be used to harness placebo effects in non-malignant pain? A literature review and survey to develop a taxonomy. BMN Open 2017;7:e015516.

18 American Medical Association: AMA Code of Medical Ethics. Opinion 8.083 - placebo use in clinical practice. 2007. https://www. ama-assn.org/delivering-care/use-placeboclinical-practice.

19 Tilburt JC, Emanuel EJ, Kaptchuk TJ, Curlin FA, Miller FG: Prescribing "placebo treatments": results of national survey of US internists and rheumatologists. BMJ 2008; 337:a1938.

20 Howick J, Bishop FL, Heneghan C, Wolstenholme J, Stevens S, Hobbs FD, Lewith G: Placebo use in the United Kingdom: results from a national survey of primary care practitioners. PLoS One 2013;8:e58247.

21 Fässler M, Meissner K, Schneider A, Linde K: Frequency and circumstances of placebo use in clinical practice - a systematic review of empirical studies. BMC Med 2010;8:15.

22 Hull SC, Colloca L, Avins A, Gordon NP, Somkin CP, Kaptchuk TJ, Miller FG: Patients' attitudes about the use of placebo treatments: telephone survey. BMJ 2013;347:f3757.
23 Fisher S, Lipman RS, Uhlenhuth EH, Rickels K, Park LW: Drug effects and initial severity of symptomatology. Psychopharmacologia 1965;7:57-60.

24 Colloca L: Treatment of pediatric migraine. $\mathrm{N}$ Engl J Med 2017;376:1387-1388.

25 Annoni M, Franklin G: Placebo effects and the ethics of therapeutic communication: a pragmatic perspective. Kennedy Inst Ethics J 2016;26:79-103.

26 Fässler M, Meissner K, Kleijnen J, Hróbjartsson A, Linde K: A systematic review found no consistent difference in effect between more and less intensive placebo interventions. J Clin Epidemiol 2015;68:442-451.

27 Blease C, Colloca L, Kaptchuk T: Are openlabel placebos ethical? Informed consent and ethical considerations. Bioethics 2016;30: 407-414.

28 Kaptchuk TJ, Friedlander E, Kelley JM, Sanchez MN, Kokkotou E, Singer JP, Kowalczykowski M, Miller FG, Kirsch I, Lembo AJ: Placebos without deception: a randomized controlled trial in irritable bowel syndrome. PLoS One 2010;5:e15591.

29 Sandler AD, Bodfish JW: Open-label use of placebos in the treatment of ADHD: a pilot study. Child Care Health Dev 2008;34:104110.

30 Carvalho C, Caetano JM, Cunha L, Rebouta P, Kaptchuk TJ, Kirsch I: Open-label placebo treatment in chronic low back pain: a randomized controlled trial. Pain 2016;157: 2766-2772.

31 Locher C, Frey Nascimento A, Kirsch I, Kossowsky J, Meyer A, Gaab J: Is the rationale more important than deception? A randomized controlled trial of open-label placebo analgesia. Pain 2017;158:2320-2328.

32 Colloca L, Howick J: Placebos without deception: outcomes, mechanisms, an ethics. Int Rev Neurobiol 2018;138:219-240.

33 Charlesworth JEG, Petkovic G, Kelley JM, Hunter M, Onakpoya I, Roberts N, Miller FG, Howick J: Effects of placebos without deception compared with no treatment: a systematic review and meta-analysis. J Evid Based Med 2017;10:97-107.

34 Kelley JM, Kaptchuk TJ, Cusin C, Lipkin S, Fava M: Open-label placebo for major depressive disorder: a pilot randomized controlled trial. Psychother Psychosom 2012;81:312314.
35 Colloca L: Tell me the truth and I will not be harmed: informed consents and nocebo effects. Am J Bioeth 2017;17:46-48.

36 Varelmann D, Pancaro C, Cappiello EC, Camann WR: Nocebo-induced hyperalgesia during local anesthetic injection. Anesth Analg 2010;110:868-870.

37 Blease C: Authorized concealment and authorized deception: well-intended secrets are likely to induce nocebo effects. Am J Bioeth 2015; 15:23-25.

38 Howe LC, Goyer JP, Crum AJ: Harnessing the placebo effect: exploring the influence of physician characteristics on placebo response. Health Psychol 2017;36:1074-1082.

39 Kaptchuk TJ, Kelley JM, Conboy LA, Davis RB, Kerr CE, Jacobson EE, Kirsch I, Schyner RN, Nam BH, Nguyen LT, Park M, Rivers AL, McManus C, Kokkotou E, Drossman DA, Goldman P, Lembo AJ: Components of placebo effect: randomised controlled trial in patients with irritable bowel syndrome. BMJ 2008;336:999-1003.

40 Koban L, Ruzic L, Wager TD: Brain predictors of individual differences in placebo responding; in Colloca L, Flaten MA, Meissner K (eds): Placebo and Pain. Elsevier/Academic Press, 2013.

41 Papakostas GI, Fava M: Does the probability of receiving placebo influence clinical trial outcome? A meta-regression of double-blind, randomized clinical trials in MDD. Eur Neuropsychopharmacol 2009;19:34-40.

42 Fava M: Implications of a biosignature study of the placebo response in major depressive disorder. JAMA Psychiatry 2015;72:10731074.

43 Blease C, Kirsch I: The placebo effect and psychotherapy: implications for theory, research, and practice. Psychol Conscious (Wash DC) 2016;3:105-107.

44 Doering BK, Rief W: Utilizing placebo mechanisms for dose reduction in pharmacotherapy. Trends Pharmacol Sci 2010;33:165-172.

45 Tekampe J, van Middendorp H, Meeuwis SH, van Leusden JW, Pacheco-López G, Hermus AR, Evers AWM: Conditioning endocrine and immune parameters in humans: a systematic review. Psychother Psychosom 2017; 86:99-107.

46 Colloca L, Enck P, DeGrazia D: Relieving pain using dose-extending placebos: a scoping review. Pain 2016;157:1590-1598. 